To appear in Proc. of the 7th IGPP International Astrophysics Conference

"Particle Acceleration and Transport in the Heliosphere and Beyond" (2008), eds. G. Li, et al. (AIP Conf. Proc., New York).

\title{
Particle Acceleration at Interplanetary Shocks
}

\author{
Matthew G. Baring \& Errol J. Summerlin \\ Department of Physics and Astronomy, MS-108, Rice University, P. O. Box 1892, \\ Houston, TX 77251-1892, USA \\ Email: baring@ rice.edu, xerex@ rice.edu
}

\begin{abstract}
The acceleration of interstellar pick-up ions as well as solar wind species has been observed at a multitude of interplanetary (IP) shocks by different spacecraft. The efficiency of injection of the pick-up ion component differs from that of the solar wind, and is strongly enhanced at highly oblique and quasi-perpendicular shock events. This paper expands upon previous work modeling the phase space distributions of accelerated ions associated with the shock event encountered on day 292 of 1991 by the Ulysses mission at 4.5 AU. As in the prior work, a kinetic Monte Carlo simulation is employed here to model the diffusive acceleration process. This exposition presents recent developments pertaining to the incorporation into the simulation of the diffusive characteristics incurred by field line wandering (FLW), according to the work of Giacalone and Jokipii. Resulting ion distributions and upstream diffusion scales are presented and compared with Ulysses data. For a pure field-line wandering construct, it is determined that the upstream spatial ramp scales are too short to accommodate the HI-SCALE flux increases for $200 \mathrm{keV}$ protons, and that the distribution function for $\mathrm{H}^{+}$somewhat underpopulates the combined SWICS/HI-SCALE spectra at the shock. This contrasts our earlier theory/data comparison where it was demonstrated that diffusive transport in highly turbulent fields according to kinetic theory can successfully account for both the proton distribution data near the shock, and the observation of energetic protons upstream of this interplanetary shock, using a single turbulence parameter. The principal conclusion here is that, in a FLW scenario, the transport of ions across the mean magnetic field is slightly less efficient than is required to effectively trap energetic ions within a few Larmor radii of the shock layer, and thereby sustain acceleration at levels that match the observed distributions. This highlights the contrast between ion transport in highly turbulent shock environs and remote, less-disturbed interplanetary regions.
\end{abstract}

Keywords: Interplanetary shocks; co-rotating interaction regions; Ulysses mission; diffusive shock acceleration; hydromagnetic turbulence

PACS: $96.50 . \mathrm{Bh}$; 96.50.Fm; 96.50.Pw; 96.50.Tf; 96.50.Vg; 96.50.Ya

\section{INTRODUCTION}

There is bountiful evidence for efficient particle acceleration at collisionless shocks in the heliosphere, and interplanetary (IP) shocks provide interesting and useful test cases for shock acceleration theories. For observations of energetic ion populations at IP shocks in the pre-Ulysses era, see, for example, [1-3]. A leading hypothesis is that in these systems, both solar wind and pick-up ions are energized to above $200 \mathrm{keV}$ by the mechanism of diffusive shock acceleration. This process forms the focus of this paper, which employs the kinematic Monte Carlo technique of Ellison and Jones (e.g., [4-6]) to model ion diffusion and convection in the turbulent shock environs, and describes the acceleration of particles that are injected directly from the thermal and pick-up ion populations. Upstream plasma quantities are input from observational data, and downstream quantities are determined using the full MHD Rankine-Hugoniot relations. The Monte Carlo method was employed by Ellison et al. [7] to perform the 
first successful theory/data comparison for the quasi-parallel portion of the Earth's bow shock, using proton, $\mathrm{He}^{++}$and other ion distributions from the AMPTE experiment.

A similar theory/data comparison was enunciated for IP shocks in Baring et al. [8], where impressive agreement was found between the simulation predictions and spectral data obtained by the Solar Wind Ion Composition Spectrometer (SWICS) aboard Ulysses, in the case of two highly-oblique shocks observed early in the Ulysses mission. Such agreement was possible only with the assumption of diffusion fairly near the Bohm limit. Summerlin \& Baring [9, 10] extended this program to explore the role of pick-up ions in the acceleration process at an IP shock detected by Ulysses' SWICS and HI-SCALE instruments at around 4.5 AU, as reported in [11]. Phase space distributions from the simulations, in the case of large angle scattering, were compared with the data, yielding acceptable fits for the proton populations and upstream flux increases, using standard prescriptions for the injected pick-up ion distribution. This paper further extends our investigative program by exploring whether or not diffusion due to field line wandering can model Ulysses' observations for this shock equally well.

\section{MODELING ULYSSES DATA FOR THE DAY 292, 1991 SHOCK}

This work thus continues our case study of the forward shock of a CIR encountered by Ulysses on Day 292 of 1991, for which downstream particle distributions were published in Gloeckler et al. [11]. Various plasma parameters for this oblique shock were input for the Monte Carlo simulation, and were obtained from [11] and the data compilations of $[12,13]$. Most important among these was the angle $\theta_{B n 1}=50^{\circ} \pm 11^{\circ}$ the upstream magnetic field made with the shock normal. This shock was also quite weak, with a sonic Mach number of $M_{\mathrm{S}} \sim 2.53$, and an inferred value [11] of $r=u_{1} / u_{2}=2.4 \pm 0.3$ for the velocity compression ratio. The normalization of solar wind proton distributions was established using $n_{p}=2.0 \mathrm{~cm}^{-3}$ as the solar wind proton density. Other parameters used in the simulation, such as the upstream flow speed of $u_{1} \approx 55 \mathrm{~km} / \mathrm{s}$ in the shock rest frame, and upstream plasma temperatures, are detailed in Summerlin \& Baring [10], as is the pick-up proton distribution input that was taken from [14] (see also [15]).

The Monte Carlo shock acceleration simulation is described in $[5,6,8,10]$. Particles are injected upstream and allowed to convect into the shock, meanwhile diffusing in space so as to effect multiple shock crossings, and thereby gain energy through the shock drift and Fermi processes. The particles gyrate in laminar electromagnetic fields, with their trajectories being obtained by solving the Lorentz force equation in the shock rest frame, in which there is, in general, a $\mathbf{u} \times \mathbf{B}$ electric field in addition to the magnetic field. The effects of magnetic turbulence are modeled by phenomenologically scattering these ions elastically in the rest frame of the local fluid flow. The simulation outputs particle fluxes and phase space distributions at any location upstream or downstream of the shock, and in any reference frame including that of the Ulysses spacecraft.

The simulation can routinely model either large-angle or small-angle scattering. At every scattering, the direction of the particle's momentum vector is deflected in the local fluid frame within some solid angle. The resulting effect is that the gyrocenter of a particle is shifted randomly by a distance of the order of one gyroradius or less in the plane orthogonal to the local field. Accordingly, cross-field diffusion emerges naturally 
from the simulation. For large angle scattering (LAS), the scattering solid angle is $4 \pi$ steradians, and the transport is governed by kinetic theory $[6,16]$, where the ratio of the spatial diffusion coefficients parallel $\left(\kappa_{\|}=\lambda v / 3\right)$ and perpendicular $\left(\kappa_{\perp}\right)$ to the mean magnetic field is given by $\kappa_{\perp} / \kappa_{\|}=1 /\left(1+\eta^{2}\right)$. Here, the parameter $\eta=\lambda / r_{g}$ is the ratio of a particle's mean free path $\lambda$ to its gyroradius $r_{g}$. Clearly, $\eta$ controls the amount of cross-field diffusion, and is a measure of the level of turbulence present in the system, i.e. is an indicator of $\langle\delta B / B\rangle$. The Bohm limit of quasi-isotropic diffusion is realized when $\eta \sim 1$ and $\langle\delta B / B\rangle \sim 1$. In LAS applications, $\eta$ is prescribed to be independent of momentum. In general, it is a parameter that critically controls the injection efficiency of low energy particles, and the upstream diffusion scale of accelerated ions.

The Monte Carlo simulation can specify arbitrary ratios of perpendicular to parallel diffusion $\kappa_{\perp} / \kappa_{\|}$in a variety of ways. The implementation in this work employed a construct that embodies the key signatures of the field-line wandering (FLW) study of Giacalone and Jokipii [17]: they found that (i) $\kappa_{\perp} / \kappa_{\|}$does not vary significantly with particle momentum, and (ii) $\kappa_{\|}=\lambda_{\|} v / 3$ is a weak function of momentum, namely $\kappa_{\|} \propto p^{1+\alpha}$ for $\alpha \sim 1 / 3$. This second property is readily incorporated in the Monte Carlo technique via a momentum-dependent parameter $\eta=\eta(p)$, i.e. $\lambda_{\|}=\eta r_{g} \propto p^{\alpha} \Rightarrow$ $\eta(p) \propto p^{\alpha-1}$, so that $\alpha \approx 1 / 3$, and $\eta(p)$ declines with increasing momentum. The approximate constancy of the ratio $\kappa_{\perp} / \kappa_{\|}$can then be accommodated by adapting the small-angle scattering implementation specified in [18]. Particle momenta are deflected uniformly within a cone of opening angle $\theta_{\text {scatt }} \ll 1$, whose axis is centered on the pre-scattering vector momentum. Note that $\kappa_{\perp}$ and $\kappa_{\|}$can be analytically coupled to field turbulence models via quasi-linear theory. Approximately $\pi / \theta_{\text {scatt }}^{2}$ deflections accumulate to effect an isotropization of the momenta. In a single scattering event with $\theta_{\text {scatt }} \ll 1$, the effective diffusive mean free path across the field is of the order of $\lambda_{\perp} \sim 2 \theta_{\text {scatt }} r_{g} / 3$. It then follows from kinetic theory that $\kappa_{\perp} / \kappa_{\|} \approx \theta_{\text {scatt }}^{2} /\left[6\left(1+\eta^{2}\right)\right]$. As $\eta \gg 1$ for most momenta, then $\theta_{\text {scatt }} \propto \eta \propto p^{\alpha-1}$ is required to achieve constancy of $\kappa_{\perp} / \kappa_{\|}$. Let the operating momentum range of particles of interest for this IP shock acceleration problem be $p_{\min } \lesssim p \leq p_{\max }$. Two global parameters are then defined for the simulation runs: $\theta_{0}=\theta_{\text {scatt }}\left(p_{\text {min }}\right)$ and $\eta_{0}=\eta\left(p_{\text {min }}\right)$ for $p_{\min }=m_{p} u_{1}$. Hence

$$
\theta_{\text {scatt }}(p)=\theta_{0}\left(\frac{p}{p_{\min }}\right)^{\alpha-1}, \eta(p)=\eta_{0}\left(\frac{p}{p_{\min }}\right)^{\alpha-1}, \quad \alpha \approx 1 / 3,
$$

then encapsulate the momentum dependences of the diffusion parallel to and perpendicular to the mean field. Field fluctuations in typical IP shocks are tantamount to magnetic turbulence variances of $\sigma^{2} \sim 0.03-0.1 B^{2}$, corresponding to ratios $\kappa_{\perp} / \kappa_{\|} \sim$ $10^{-4}-10^{-3}$ according to the field line wandering results in Figure 4 of [17]. This then sets $\theta_{0} / \eta_{0} \sim 10^{-2}-10^{-1}$ for the FLW-initiated spatial diffusion modeling here. One final nuance must be identified. The cumulative effect of $\sim \pi / \theta_{\text {scatt }}^{2}$ deflections does not amount to $\kappa_{\|}=\eta r_{g} v / 3$ per se, unless the vector momentum is more or less reversed on the $\lambda_{\|}$scale. Hence, the implementation of FLW-instigated spatial diffusion here imposes an approximate reversal of the direction of the component of momentum parallel to $\mathbf{B}$ every scattering event. Physically this amounts to a magnetic mirroring event, as is implied by the FLW study of [17], superposed on a small amount of isotropic diffusion. 

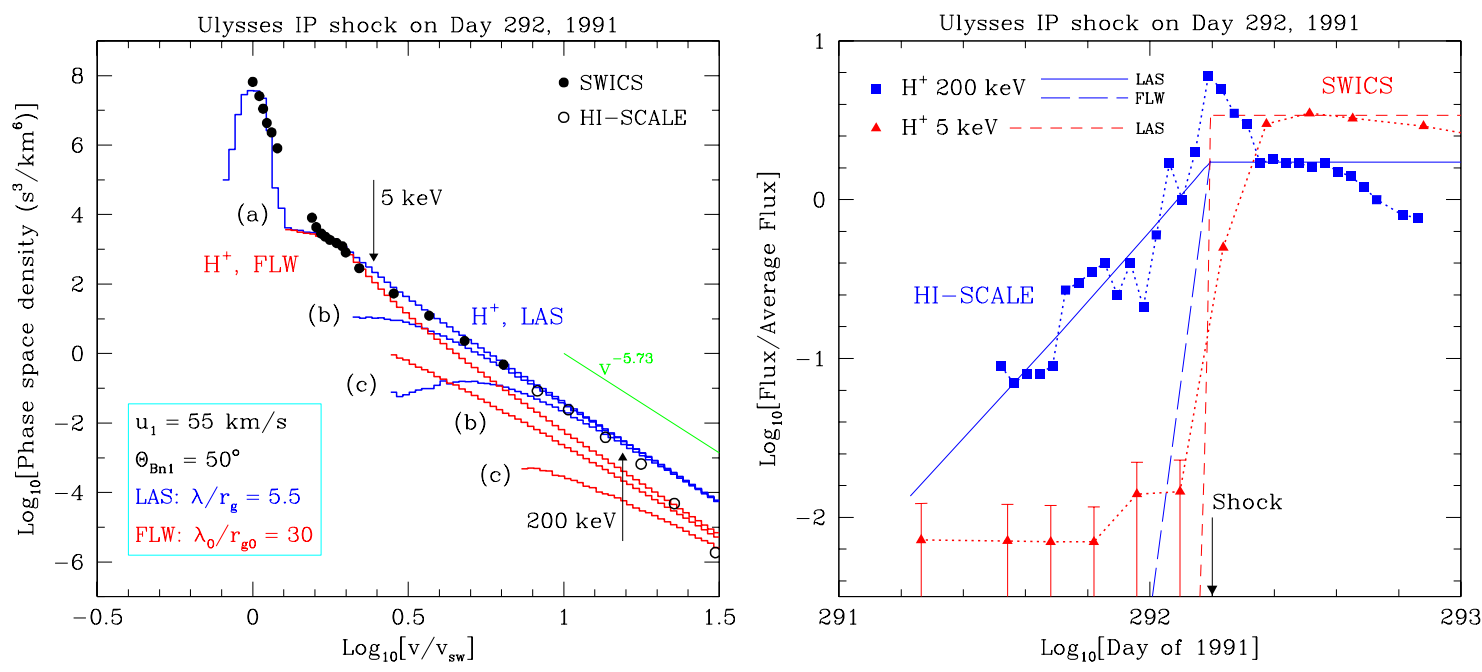

FIGURE 1. Left panel: Comparison between phase space velocity distributions for downstream data collected by the Ulysses mission for the shock on day 292 of 1991, and Monte Carlo model results. The data are for $\mathrm{H}^{+}$solar wind and pickup ions (filled circles for SWICS data; open circles for HI-SCALE points), and are taken from Gloeckler et al. [11]. The heavyweight histograms are the corresponding Monte Carlo models of acceleration of protons for $u_{1}=55 \mathrm{~km} / \mathrm{sec}$, using plasma shock parameters from [11] and sources indicated in the text (see also [10]). Two model cases are depicted - the blue (labeled $H^{+}$, LAS) denotes a large angle scattering implementation with $\eta=\lambda / r_{g}=5.5$, and the red (labeled $H^{+}$, FLW) corresponds to a field line wandering diffusive model with $\eta_{0} \equiv \lambda_{0} / r_{g 0}=30$. In each case, there are three spectra shown, corresponding to (a) just downstream, and successively increasing times upstream of the shock encounter, i.e. (b) 14 minutes and (c) 69 minutes. The velocity axis is the ratio of the ion speed $v$, as measured in the spacecraft frame, to the solar wind speed $v_{s w}$. The shock compression ratio was $r=2.1$, implying diffusive acceleration power-laws of index -5.73 , indicated in green.

Right panel: The flux variations of accelerated pick-up ion populations as a function of time near the shock. The data for $5 \mathrm{keV}$ and $200 \mathrm{keV}$ pick-up $\mathrm{H}^{+}$are depicted by filled red triangles and blue squares, respectively, and are taken from [11]. The Monte Carlo model generated fluxes at different distances normal to the shock, which were converted to spacecraft times by incorporating solar wind convection. The $5 \mathrm{keV}$ and $200 \mathrm{keV}$ pick-up $\mathrm{H}^{+}$LAS model traces are displayed as red dashed and blue solid curves, respectively, and exhibit an exponential decline upstream of the shock that is characteristic of diffusive shock acceleration. The $200 \mathrm{keV}$ FLW model profile (blue, long dashes) displays an upstream ramp that is much shorter than the observed profile, due to low $\lambda / r_{g}$ values at these high ion energies.

Results for the acceleration of solar wind and pick-up ions for the case of large angle scattering in strong field turbulence $(\langle\delta B / B\rangle \gtrsim 0.1)$ were presented and discussed at length in $[9,10]$. A selection of these are exhibited in Figure 1, which displays downstream distributions for thermal, pick-up and accelerated protons from the Monte Carlo simulation, and the SWICS and HI-SCALE measurements (see Fig. 1 of [11]) taken in the frame of the spacecraft on the downstream side of the Day 292, 1991 shock. The solar wind and pick-up proton parameters are fairly tightly specified, so that the ratio of the particle mean free path to its gyroradius, $\eta=\lambda / r_{g}$ is essentially the only free model parameter. The efficiency of acceleration of low energy ions in oblique shocks, i.e. the normalization of the non-thermal power-law, is sensitive $[6,8]$ to $\eta$, which was adjusted to obtain a reasonable "fit" to the data. For the large angle scattering case, the downstream fit in the left hand panel of Fig. 1 models the accelerated protons well for 
a rigidity-independent $\eta=5.5 \pm 1.5$, a value consistent with a moderate level of field turbulence; the uncertainty in $\eta$ is due mostly to the observational uncertainty in the shock obliquity $\theta_{B n 1}$. The non-thermal proton distribution is composed virtually entirely of accelerated pick-up ions that are energized much more efficiently than thermal solar wind $\mathrm{H}^{+}$ions. Note that the distribution of accelerated $\mathrm{He}^{+}$pick-up ions reported by [11] for this shock can be modeled $[9,10]$ by the same scattering parameter $\eta=5.5$.

The left hand panel of Figure 1 also displays results for the field-line wandering implementation as prescribed in Eq. (1), for the specific case of $\eta_{0}=30$ and $\theta_{0}=0.74$. This case approximates a $\kappa_{\perp} \sim 10^{-4} \kappa_{\|}$scenario, i.e. a variance of $\sigma^{2} \sim 0.03 B^{2}$ for the GJ99 turbulence analysis. The key conclusion is that the FLW model underpredicts the Ulysses data downstream, largely because the retention of ions in the shock layer is basically less efficient than in the LAS diffusion picture. Trial runs with higher values of $\eta_{0}$ and $\theta_{0}$ improve this situation somewhat, but not sufficiently to match the observations. This is a generic difficulty for the FLW implementation, that can be truly rectified only for larger ratios $\kappa_{\perp} / \kappa_{\|}$and variances $\sigma^{2}$. Such turbulent conditions are more commensurate with conditions in IP shocks than the low variance constructs that were employed in GJ99 that are appropriate to the quiet interplanetary solar wind. A fuller exploration of such turbulence parameter space will be performed in future work.

As highlighted in [10], an informative diagnostic on the acceleration model is to probe the spatial scale of diffusion upstream of the shock. Results are illustrated in the left hand panel of Fig. 1 via the display of upstream distributions of high energy particles at different times, i.e. distances from the shock. These exhibit a characteristic "peel-off" effect [19] where superthermal ions become depleted at successively high energies the further the detection plane is upstream. Fluxes for two different $H^{+}$ion energies, $5 \mathrm{keV}$ and $200 \mathrm{keV}$, were obtained from spectra like those in the left hand panel in the Fig. 1, and are displayed in the right hand panel of the Figure, together with corresponding data from Fig. 3 of [11] for identical energy windows. The normalization of the Ulysses data was established by averaging over 3 day intervals, whereas the model normalization was adjusted to match observed fluxes around half a day downstream of the shock.

As particles diffuse upstream of the shock against the convective flow, high energy ions with a mean free path $\lambda \propto r_{g}^{\alpha}$ establish an exponential dilution in space/time upstream. For the LAS case with $\alpha=1$, this spatial scale of the exponential decline is more or less identical to that of the model (modulo plasma fluctuations) for the same choice of $\eta=5.5$ that optimized the spectral agreement. This attractive concordance was featured in [10], and is highly suggestive that ion diffusion in strong field turbulence that is intimately connected to the shock is an integral part of the acceleration process. In contrast, Fig. 1 indicates that the diffusive scale upstream for the $\alpha=1 / 3, \eta_{0}=30$ FLW model is far too short for $200 \mathrm{keV}$ ions to accommodate the data. This principally arises because the dominant contribution to the diffusion length is $\ell \sim \kappa_{\|} \sin ^{2} \theta_{B n 1} / u_{1}$, which is much smaller at $200 \mathrm{keV}$ energies than in the LAS case, by virtue of the form in Eq. (1). Preliminary runs indicate that, for the FLW construction, while $\ell$ can be increased to match the observations, it is more difficult to model the spectra than in the LAS scenarios that describe highly turbulent $\langle\delta B / B\rangle \gtrsim 0.1$ regimes. While the LAS simulation results are consistent with the observed results, it is impossible to draw more definitive conclusions discriminating between LAS and FLW models, without a focus on ions of intermediate energy, say around 50-100 keV, the subject of future work. 


\section{CONCLUSIONS}

This paper has modeled measurements of the phase space distributions for protons observed by the Ulysses instruments SWICS and HI-SCALE in the Day 292, 1991 shock using a Monte Carlo simulation of diffusive shock acceleration. Two diffusion models were employed, large angle scattering appropriate for highly turbulent regions, and one approximating the characteristics of field line wandering as determined by Giacalone and Jokipii [17]. In both, the injection of pick-up protons dominates that of solar wind protons in this highly oblique shock. It was found that while there was consistency between the LAS model and the data for energetic protons above speeds around 600 $\mathrm{km} / \mathrm{sec}$, outlined at length in [10], the FLW scenario explored here has some difficulty in generating superthermal $\mathrm{H}^{+}$in the power-law domain with the requisite efficiency. This is attributed to the comparative lack of cross-field diffusion $\left(\kappa_{\perp} / \kappa_{\|} \lesssim 10^{-3}\right)$ in the FLW model, which is implemented for variances $\sigma^{2} \lesssim 0.1 B^{2}$ commensurate with field data for the quiet, interplanetary solar wind. Because a decline of $\lambda / r_{g}$ with ion momentum is implicit in the FLW model, upstream ramp diffusion scales are accordingly very short at $200 \mathrm{keV}$ energies, much shorter than the observed pre-shock scale in HI-SCALE data, unless large values of $\eta_{0} \equiv \lambda_{0} / r_{g 0} \gtrsim 130$ for suprathermal ions are adopted. Summerlin $\&$ Baring [10] observed that the LAS model could consistently explain this upstream ramp. Investigation of the turbulence parameter space in the FLW implementation is ongoing, to assess whether it absolutely requires higher $\kappa_{\perp} / \kappa_{\|}$in order to successfully model the data. However, the fact that the diffusive LAS model works so well in coupling the spectral and spatial properties suggests that diffusion in highly turbulent fields is an integral aspect of the acceleration process at this shock.

\section{REFERENCES}

1. Sarris, E. T., \& Van Allen, J. A. 1974, J. Geophys. Res. 79, 4,157.

2. Decker, R. B., Pesses, M. E., \& Krimigis, S. M. 1981, J. Geophys. Res. 86, 8819.

3. Tan, L. C., Mason, G. M., Gloeckler, G., \& Ipavich, F. M. 1988, J. Geophys. Res. 93, 7,225.

4. Ellison, D. C., Jones, F. C. \& Eichler, D. 1981, J. Geophys. - Zeitschrift fuer Geophysik, 50, 110.

5. Jones, F. C. \& Ellison, D. C. 1991, Space Sci. Rev. 58, 259.

6. Ellison, D. C., Baring, M. G. \& Jones, F. C. 1995, ApJ453, 873.

7. Ellison, D. C., Möbius, E., \& Paschmann, G. 1990, ApJ 352, 376.

8. Baring, M. G., Ogilvie, K. W., Ellison, D., \& Forsyth, R. 1997, ApJ 476, 889.

9. Baring, M. G., \& Summerlin, E. J. 2005, in Proc. 4th IGPP International Astrophysics Conference, eds. G. Li, et al. (AIP Conf. Proc. 781, New York), p. 207.

10. Summerlin, E. J. \& Baring, M. G. 2006, Adv. Space Res. 38(7), 1281.

11. Gloeckler, G., Geiss, J., Roelof, E. C., et al. 1994, J. Geophys. Res.99, 17,637.

12. Balogh, A., et al. 1995, Space Sci. Rev.72, 171.

13. Hoang, S., et al. 1995, Adv. Space Res. 15 (8/9), 371. Adv. Space Res. 38(7), 1281.

14. Ellison, D. C., Jones, F. C. \& Baring, M. G. 1999, ApJ 512, 403.

15. le Roux, J. A., Potgieter, M. S., \& Ptuskin, V. S. 1996, J. Geophys. Res. 101, 4,791.

16. Forman, M. A., Jokipii, J. R. \& Owens, A. J. 1974, ApJ 192, 535.

17. Giacalone, J., \& Jokipii, J. R. 1999, ApJ 520, 204.

18. Ellison, D. C., Jones, F. C. \& Reynolds, S. P. 1990, ApJ 360, 702.

19. Lee, M. A. 1982, J. Geophys. Res. 87, 5063. 